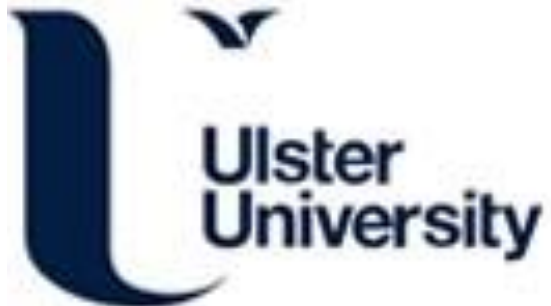

\section{Assisted Standing for Duchenne Muscular Dystrophy}

Pedlow, K. (2019). Assisted Standing for Duchenne Muscular Dystrophy. Cochrane Database of Systematic Reviews, 2019(10), [CD011550]. https://doi.org/10.1002/14651858.CD011550.pub2

Link to publication record in Ulster University Research Portal

\section{Published in:}

Cochrane Database of Systematic Reviews

Publication Status:

Published (in print/issue): 13/10/2019

DOI:

10.1002/14651858.CD011550.pub2

\section{Document Version}

Publisher's PDF, also known as Version of record

\section{General rights}

Copyright for the publications made accessible via Ulster University's Research Portal is retained by the author(s) and / or other copyright owners and it is a condition of accessing these publications that users recognise and abide by the legal requirements associated with these rights.

\section{Take down policy}

The Research Portal is Ulster University's institutional repository that provides access to Ulster's research outputs. Every effort has been made to ensure that content in the Research Portal does not infringe any person's rights, or applicable UK laws. If you discover content in the Research Portal that you believe breaches copyright or violates any law, please contact pure-support@ulster.ac.uk. 
Cochrane Database of Systematic Reviews

\section{Assisted standing for Duchenne muscular dystrophy (Review)}

Pedlow K, McDonough S, Lennon S, Kerr C, Bradbury I

Pedlow K, McDonough S, Lennon S, Kerr C, Bradbury I.

Assisted standing for Duchenne muscular dystrophy.

Cochrane Database of Systematic Reviews 2019, Issue 10. Art. No.: CD011550.

DOI: 10.1002/14651858.CD011550.pub2.

www.cochranelibrary.com 
TABLE OF CONTENTS

HEADER 1

ABSTRACT

PLAIN LANGUAGE SUMMARY

BACKGROUND

OBJECTIVES

METHODS

Figure 1.

RESULTS

DISCUSSION

AUTHORS' CONCLUSIONS

ACKNOWLEDGEMENTS

REFERENCES

CHARACTERISTICS OF STUDIES

APPENDICES

CONTRIBUTIONS OF AUTHORS

DECLARATIONS OF INTEREST

SOURCES OF SUPPORT

DIFFERENCES BETWEEN PROTOCOL AND REVIEW 
[Intervention Review]

\section{Assisted standing for Duchenne muscular dystrophy}

Katy Pedlow ${ }^{1,2}$, Suzanne McDonough 2,3 , Sheila Lennon ${ }^{4}$, Claire Kerr ${ }^{5}$, Ian Bradbury 6

${ }^{1}$ Centre for Health and Rehabilitation Technologies (CHaRT), Institute of Nursing and Health Research, Ulster University, Newtownabbey, UK. 2Physiotherapy, Royal College of Surgeons in Ireland, Dublin, Ireland. ${ }^{3}$ Centre for Health and Rehabilitation Technologies (CHaRT), Institute of Nursing and Health Research, Ulster University, Newtownabbey, UK. 4Physiotherapy, College of Nursing and Health Sciences, Flinders University, Adelaide, Australia. ${ }^{5}$ School of Nursing \& Midwifery, Queen's University Belfast, Belfast, UK. ${ }^{6}$ Frontier Science Scotland, Kincraig, UK

Contact address: Katy Pedlow, Centre for Health and Rehabilitation Technologies (CHaRT), Institute of Nursing and Health Research, Ulster University, Room 1F110, Shore Road, Newtownabbey, County Antrim, BT37 OQB, UK. k.pedlow@ulster.ac.uk.

Editorial group: Cochrane Neuromuscular Group

Publication status and date: New, published in Issue 10, 2019.

Citation: Pedlow K, McDonough S, Lennon S, Kerr C, Bradbury I. Assisted standing for Duchenne muscular dystrophy. Cochrane Database of Systematic Reviews 2019, Issue 10. Art. No.: CD011550. DOI: 10.1002/14651858.CD011550.pub2.

Copyright ( 2019 The Cochrane Collaboration. Published by John Wiley \& Sons, Ltd.

\section{A B S T R A C T}

\section{Background}

Duchenne muscular dystrophy (DMD) is the most common X-linked neuromuscular disorder. When boys with DMD reach the second decade of life, they lose their ability to walk and become wheelchair dependent. Standing devices and orthoses are considered to be an essential component in the therapy management of DMD. Clinical opinion and research from other neurological conditions highlight the proposed benefits of standing device use, however, its effect within this population is currently unknown. A review of the evidence for the use of standing devices and orthoses is necessary to inform all stakeholders, including people with DMD, clinicians, decision makers and funders, and to guide future research.

\section{Objectives}

To assess the effects of standing devices and orthoses on musculoskeletal impairments (such as pain, contracture, scoliosis development and bone density) in boys and men with DMD, and secondarily to determine their effect on quality of life, participation in activities, and patient experience (satisfaction). We also considered any adverse events associated with their use.

\section{Search methods}

We searched the Cochrane Neuromuscular Specialised Register, CENTRAL, MEDLINE, Embase, AMED, PsycINFO, CINAHL Plus, PEDro, and ProQuest Dissertations \& Theses Global up to 5 September 2019. We checked references in identified trials, handsearched journal abstracts, and searched trials registries.

\section{Selection criteria}

We planned to include randomised controlled trials (RCTs) and quasi-RCTs of any model of standing device for use in DMD. The control interventions would have been any other comparison group, including no standing device, a different model of standing device, usual care, or an alternative form of assistive weight bearing.

\section{Data collection and analysis}

We used standard Cochrane methodological procedures.

\section{Main results}

Although we identified 13 potentially relevant studies, none met the inclusion criteria for this review. 


\section{Authors' conclusions}

Since there were no RCTs or quasi-RCTs available to evaluate the effectiveness of standing devices in people with DMD, studies are needed to investigate the effectiveness of standing devices in this population.

\section{PLAIN LANGUAGE SUMMARY}

The use of standing devices for boys with Duchenne muscular dystrophy

\section{Review question}

What is the effect of using standing devices in boys with Duchenne muscular dystrophy (DMD)?

\section{Background}

DMD is a condition that affects only boys. The progressive muscle wasting that occurs with the condition results in decreased walking ability and eventually, use of a wheelchair for mobility. Specialist equipment is used routinely to assist boys with DMD to stand or bear weight. Such equipment includes standing devices (e.g. frames or standing wheelchairs) and orthoses (e.g. braces or splints). We have evidence from other groups of people that standing reduces physical problems, including pain, development of contractures (fixed shortening of muscles) and curvature of the spine. Standing also strengthens bones. However, we have no evidence about the effects of the use of standing frames or orthoses by boys and men with DMD.

We searched the medical literature for studies that met our inclusion criteria. We required studies to have assessed standing devices for use in DMD compared to no standing device, a different model of standing device, usual care, or an alternative aid to enable weight bearing. Studies had to randomly allocate participants to treatment groups (these studies usually provide the highest quality evidence).

\section{Key results and quality of evidence}

We found no studies that met our inclusion criteria.

\section{Conclusion}

We found no relevant trials eligible for this review to determine the effectiveness of standing devices for boys with DMD. We need further studies to determine whether standing devices have an impact on specific health problems, participation in activities, and quality of life. Future research studies should also focus on the 'dose' of standing (i.e. the duration, frequency and amount of weight bearing) and the use of different orthoses.

The evidence is current to September 2019. 


\section{B A C K G R O U N D}

\section{Description of the condition}

Duchenne muscular dystrophy (DMD) is the most common X-linked neuromuscular disorder, affecting approximately 1 in 3300 to 6000 live male births (Deconinck 2007; Mendell 2012). The underlying causes are deletions, duplications and mutations in the dystrophin gene. With recent advances in treatment, boys with this life-limiting condition are now living into their fourth or even fifth decades. By contrast, few boys with DMD survived beyond their early twenties in the past. Although boys may show symptoms of the condition at an early stage, most are diagnosed around the age of five years. Formal diagnosis of DMD is typically through a combination of methods, including a clinical diagnosis of progressive muscle weakness, family history and increased levels of creatine kinase in the blood (Birnkrant 2018a). To provide a definitive diagnosis, genetic testing via blood sampling is often performed, with or without a muscle biopsy (Manzur 2008).

The predominant clinical presentation is wasting of the proximal muscles, for example of the trunk, shoulder girdle, or both. Symptoms at presentation are either a delay in reaching typical motor milestones (e.g. walking), or signs such as toe walking, poor motor skills, frequent falls, difficulty climbing stairs and a waddling gait (Birnkrant 2018b). Fatigue and learning difficulties may also be present. Although symptoms are often initially mild, there is a rapid physical and functional decline due to progressive muscle wasting (Kohler 2009). Once boys reach the second decade of life, they lose their ability to walk, and become wheelchair dependent (Kinali 2007). At this time, a number of significant and complex secondary comorbidities are often associated with the condition. These include the development of muscle contractures, osteoporosis, scoliosis, weight gain and respiratory complications, such as impaired pulmonary function (Birnkrant 2018b). All of these comorbidities can potentially be managed. Effective management of the condition and its comorbidities is required to enhance quality of life for these children and their families.

\section{Description of the intervention}

Assisted standing involves using a device to help place load through a person's feet. Boys and men with DMD achieve assisted standing via two main methods: standing devices (including standing frames, standing wheelchairs and tilt tables) and orthoses. For the purposes of this review, orthoses will only include hip-knee-ankle-foot orthosis (HKAFO), knee-ankle-foot orthoses (KAFO), and standing shells. The two methods can be used both within the therapy setting and in daily life in school or at home. Standing devices and orthoses provide a stable mechanical support for weight bearing in supine, prone or upright positions, depending on the device chosen; however, a precise and timely evaluation of individual needs is required to select the most appropriate design of standing device, orthoses, or both. One survey of therapists reported that $68 \%$ of them prescribed standing frames for people in the nonambulant stage of DMD (Eagle 2002). Traditionally, assisted standing devices are prescribed for non-ambulant DMD. However, their use has been studied in both ambulant and non-ambulant children aged 5 to 15 years of age (Taktak 1995).

Although there is a lack of conclusive evidence for the use of standing devices in DMD, clinical opinion and research from other neurological conditions (such as cerebral palsy), highlight the proposed benefits (Caulton 2004; Pin 2007; Rivi 2014). These benefits include preservation of muscle length and range of joint movement via the stretch that occurs during standing (predominately of the hip and lower-limb muscles) (Gibson 2009), delayed onset of scoliosis, increased bone density (thereby reducing the risk of fractures) (Pope 2007), fewer muscle spasms and better respiratory function (including voice control) (Glickman 2010). Research on standing for other conditions has also suggested improved circulation, digestion (Paleg 2008), and bowel and bladder function (Taylor 2009).

Clinical opinion on standing indicates other benefits, including pressure relief (which improves skin integrity), improvement of well-being and better sleep. However, these reported benefits have not been systematically identified.

Standing devices are considered to be an essential component of the therapy management of DMD (both physiotherapy and occupational therapy). Various other therapy techniques for DMD are used alongside standing devices and are supported by research, including massage and stretching and the use of adaptive equipment as the disease progresses (Birnkrant 2018b). The use of standing devices significantly increases once the ability to walk has been lost, in order to maintain standing (Umphred 2013).

\section{Access to the intervention}

Access to neuromuscular services varies greatly depending upon the person's location and age (paediatric versus adult services). Physiotherapy and standing devices are typically provided during childhood, but these services may now be required throughout adolescence and adulthood, due to the increased life expectancy of people with DMD.

\section{How the intervention might work}

\section{Standing devices (supine standing frame, prone standing frame, upright standing frame, tilt table, and standing wheelchair)}

A supine standing frame requires the person to lie down on the frame before it is moved into a more upright position. The clinical advantage of a standing frame is its use with those who lack anti-gravity strength in their upper trunk, in addition to a reduction in the amount of weight bearing. A prone standing frame places the person in a forward tilt. This position encourages head control and anti-gravity use of muscles in the upper trunk. A further clinical advantage of this frame is the ability to alter the amount of weight bearing by changing the angle of tilt of the frame. This is important for people with reduced leg strength or contractures. Providing an angle of tilt reduces the amount of leg strength and joint range of movement required to maintain the position. An upright standing frame places the person in an almost vertical position to reflect a natural standing position. This has the benefit of achieving maximum weight bearing, which is important for bone density (Umphred 2013).

A tilt table is very similar to a supine standing frame; however, the person is placed on a large flat mat with straps across the chest, hips and knees, rather than the more flexible fit of a supine standing frame. A tilt table provides similar clinical benefits to a supine standing frame, but is less likely to be used in a home or classroom setting because of its size. 
A standing wheelchair allows the person to complete functional tasks in a standing position. The wheelchair can be either manually or power operated, and the height at which the person stands can be adapted depending on the patient's current standing ability, taking into account any reduction in muscle length or range of joint motion.

\section{Orthoses}

A HKAFO is a device that supports the entire lower limb. This is achieved by providing support points at the hip, knee, ankle and foot by either a metal or plastic design, in addition to a soft material, for example leather, which is used for point-of-skin contact.

A KAFO is a device that supports the knee, ankle and foot with the aim of maintaining alignment in the lower limb. In a similar manner to the HKAFO, the KAFO is shaped to match the person's limb, either with a metal or plastic design and a soft material. These orthoses are reported to increase the length of time assisted walking and standing can be maintained in DMD (Bakker 2000).

Standing shells are more commonly used by people with cerebral palsy, to create optimal alignment of the body. A plaster cast is developed to encase the legs and back. A polyethylene plastic mould is then created from the plaster cast. The plastic mould (standing shell) is placed onto the person and secured in place by leather straps. The standing shell allows people to complete functional tasks in a standing position.

Variation in standing device and orthoses design and selection is thus apparent, as is variation in the length of time spent using the device. Currently there is insufficient evidence to determine the optimal dose for standing with standing devices and orthoses.

The presence of orthostatic hypotension, healing fractures and severe contractures can prevent the use of standing devices (Holland 2006).

\section{Potential limitations of the intervention}

In this population, although there are many potential benefits to using standing devices, it is important to recognise that there may also be a number of possible disadvantages. These include the time and commitment required on the part of the person and their family to complete the intervention, and the possible associated financial costs to both the family and service provider, in terms of provision of equipment and adequately trained staff. Clinical disadvantages associated with the use of standing devices include hypotension and boredom.

One aspect in determining the potential use of standing frames is the degree of lower-limb contractures. Standing devices cannot typically accommodate severe flexion contractures of the lower limbs; additionally, weight bearing through the feet would result in excessive abnormal forces being exerted through the lower limbs, thus making a supported-standing programme inappropriate. Contractures are more likely to be severe in individuals who have been long-term wheelchair users, due to the length of time spent in sitting postures and the consequent increased risk of contracture of the hip flexor and hamstring musculature.

\section{Why it is important to do this review}

Despite clinical descriptions of the potential benefits of standing devices and orthoses for this population and research in other pop- ulations, there is no standardised approach to use of these devices in people with DMD, and a lack of unequivocal evidence of their effectiveness (Eagle 2002). An expert consensus on the management of DMD commented that the use of standing devices and orthoses is logical, but not evidenced, and highlighted that there is no consensus regarding how long they should be used for, or how frequently (Dooner 2007).

One main issue in obtaining evidence for the use of standing devices is that they may be used for many different reasons, so studies may focus on different potential benefits, adding to the complexity of achieving consensus on their use. It is also necessary to assess whether the potential advantages of assisted standing outweigh the previously described potential disadvantages in terms of time, costs and clinical outcomes.

There is a clear need to review the evidence for the use of standing devices and orthoses in order to inform all stakeholders better, including boys and men with DMD, clinicians, decision makers and funders, and to guide future research.

\section{O B J E C T IVES}

To assess the effects of standing devices and orthoses on musculoskeletal impairments (such as pain, contracture, scoliosis development, and bone density) in boys and men with DMD, and secondarily to determine their effect on quality of life, participation in activities, and patient experience (satisfaction). We also considered any adverse events associated with their use.

\section{METHODS}

\section{Criteria for considering studies for this review \\ Types of studies}

We considered randomised controlled trials (RCTs) and quasi-RCTs for inclusion. We included studies reported in full text, published as an abstract only, or unpublished. We included data from non-randomised studies in the discussion, but not in the results section of the review. We placed no restrictions on date or language of publication.

\section{Types of participants}

We included both men and boys of any age with a confirmed diagnosis of DMD.

If a study had included only a subset of participants with DMD, we would have included it only if it measured the primary outcome for the DMD subset.

\section{Types of interventions}

We considered trials that compared any model of standing device or orthoses (HKAFO, KAFO or standing shell) with any other comparison group, including no standing device, a different model of standing device (for example a tilt table versus a standing frame), usual care, or an alternative form of assistive weight bearing. Standing devices included all types of standing frames, tilt tables and standing wheelchairs. We considered the following orthotic devices: HKAFO, KAFO and standing shells. We included any trial that compared one model of standing modality to another. We allowed co-interventions in cases where they were provided to each group equally. 


\section{Types of outcome measures}

We did not use the outcomes listed here as eligibility criteria for this review, but identified them as outcomes of interest within included studies.

\section{Primary outcomes}

1. Change in range of joint movement (goniometry; Pandya 1985) in the short term (baseline to 3 months), medium term (3 to 12 months) and long term (over 12 months).

\section{Secondary outcomes}

1. Musculoskeletal impairment: measured using validated scales in the short term (baseline to 3 months), medium term (3 to 12 months) and long term (over 12 months). Musculoskeletal impairment included pain (visual analogue scale (VAS); McCormack 1988).

2. Scoliosis: measured by scoliometer (Bunnell 1984), or by Cobb's angle on X-ray (Wills 2007). Change in value from baseline to over 12 months.

3. Bone density: measured by duel-energy X-ray absorptiometry (DEXA) of the lumber spine, femoral heads, or both (Mazess 1990); change in value from baseline to over 12 months.

4. Respiratory function: measured as per cent predicted forced vital capacity (FVC\%); change in value from baseline to 3 to 12 months (Phillips 2001).

5. Participation: measured using validated scales such as Children's Assessment of Participation and Enjoyment (CAPE) (King 2004); change in baseline value in the short term (3 months), medium term ( 3 to 12 months) and long term (over 12 months).

6. Quality of life: measured using validated scales such as the Individualized Neuromuscular Quality of Life questionnaire (INQoL) (Vincent 2007), or Child Health Questionnaire (CHQ) (Landgraf 1999); measured by change in value in the short term (3 months), medium term ( 3 to 12 months) and long term (over 12 months).

7. Bowel function: measured by frequency of episodes of constipation (Lo Cascio 2014); measured by change in value in medium term (from baseline to 3 to 12 months).

8. Participant and family satisfaction with the intervention: as sessed, for example, by self-report questionnaires; measured by change of value from baseline in the short term ( 3 months), medium term ( 3 to 12 months) and long term (over 12 months). We did not include qualitative reporting of participant satisfaction.

9. Adverse events: subdivided into any adverse events, adverse events that led to discontinuation of treatment, and serious adverse events (events that were fatal, life threatening, or required prolonged hospitalisation).

\section{Search methods for identification of studies}

\section{Electronic searches}

The Trials Search Co-ordinator searched the following databases (detailed strategies are in the appendices):

1. the Cochrane Neuromuscular Specialised Register (Cochrane Register of Studies (CRS Web) 5 September 2019 (CRS Web; Appendix 1);

2. the Cochrane Central Register of Controlled Trials (CENTRAL) 5 September 2019 (CRS Web; Appendix 2);

3. MEDLINE Ovid (January 1966 to 4 September 2019; Appendix 3);

4. Embase Ovid (1947 to 4 September 2019; Appendix 4);

5. CINAHL Plus EBSCOhost (Cumulative Index to Nursing and Allied Health Literature; 1937 to 4 September 2019; Appendix 5);

6. AMED Ovid (Allied and Complementary Medicine; 1985 to August week 4 2019; Appendix 6);

7. PsycINFO Ovid (1806 to August 2019; Appendix 7);

8. ProQuest Dissertations \& Theses Global (1861 to August 2019; Appendix 8).

We also searched the Physiotherapy Evidence Database (PEDro; 1929 to September 2019; Appendix 9), the United States National Institutes of Health Clinical Trials Registry (www.ClinicalTrials.gov; Appendix 10), and the World Health Organization International Clinical Trials Registry Portal (apps.who.int/trialsearch/; Appendix 10).

We searched all databases from their inception to the present, and imposed no restriction on language of publication.

We identified non-randomised studies for inclusion in the discussion from the same search results.

\section{Searching other resources}

We searched reference lists of all primary studies and review articles for additional references. We search relevant manufacturers' websites for trial information (Higgins 2011).

We searched grey literature to identify other studies.

\section{Data collection and analysis}

\section{Selection of studies}

Two review authors (KP, SMcD) independently screened titles and abstracts of all the studies identified as a result of the searches, and coded them as 'retrieve' (eligible or potentially eligible/unclear) or 'do not retrieve'. We retrieved full-text study reports for potentially eligible studies. Two review authors (KP, SMCD) independently screened the full texts to identify studies eligible for inclusion, and identified and recorded reasons for exclusion of the ineligible studies. There were no disagreements between the authors. We reported the selection process in sufficient detail to complete a PRISMA flow diagram (Figure 1) and 'Characteristics of excluded studies' table. 
Figure 1. Study flow diagram.

\section{0 results from searches of clinical trials registries}

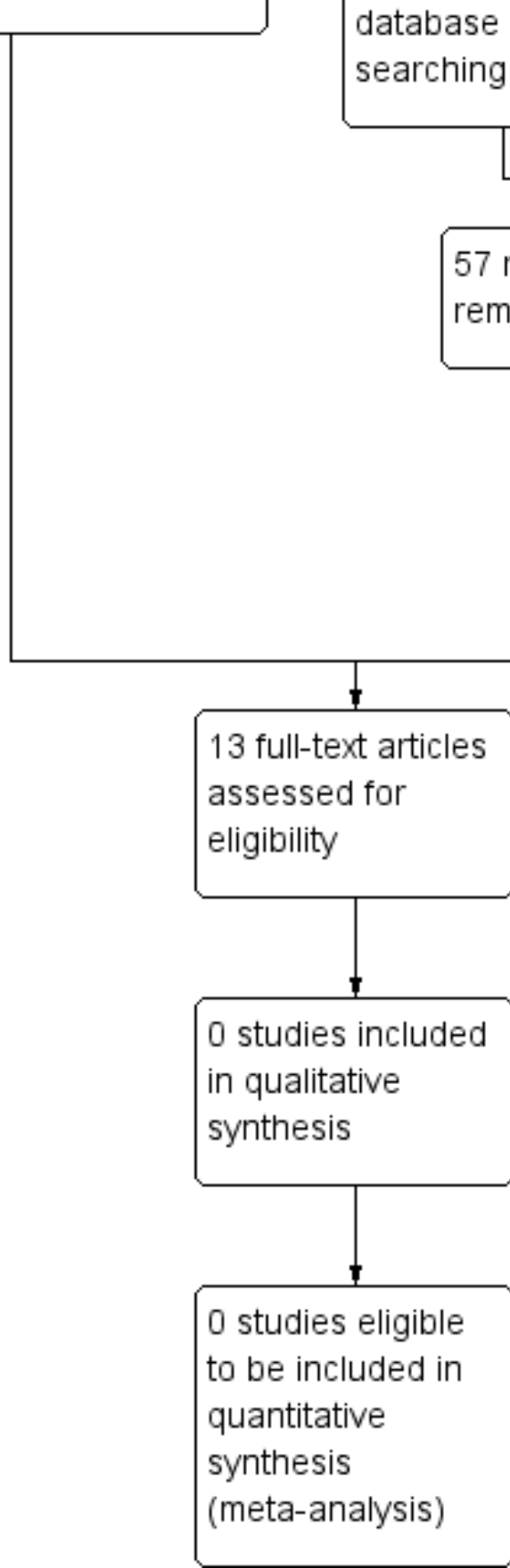

No studies met the inclusion criteria for this review.

Appendix 11 describes the data collection methods we will use, should studies become available in future.
13 full-text articles

excluded, with

reasons

\section{1 additional records identified through other sources}

7 records after duplicates removed

\begin{tabular}{|l}
57 records \\
screened
\end{tabular}$\longrightarrow \begin{aligned} & 44 \text { records } \\
& \text { excluded }\end{aligned}$

\section{RESULTS}

\section{Description of studies}

\section{Results of the search}

The search identified 90 citations (Figure 1). After duplicate removal, we screened the titles and abstracts of 57 citations and excluded 44 records. We excluded studies if they were not RCTs or quasi-RCTs, if they described surgical interventions, or if DMD was 
not the study population. We obtained 13 full-text studies, as it was unclear from their titles and abstracts whether or not they met the study criteria. All of these studies were non-RCTs; nine were cohort studies, one was a qualitative study, one was a review paper, one was a case series, and one was a consecutive sample. Furthermore, most did not have a control group for comparison. We found no ongoing studies. After assessment of the full-text reports, we found no studies that met the criteria for this review. Searches of the trials registries retrieved no records.

\section{Included studies}

No studies met the review's inclusion criteria.

\section{Excluded studies}

We have listed all 13 papers reviewed in full text as excluded studies, as they did not meet the inclusion criteria (Characteristics of excluded studies). Eleven of the studies did not focus on standing or include at least one outcome of interest. Two studies were intervention studies with a minimum of one outcome of interest (Taktak 1995; Townsend 2016). We have described them in the Agreements and disagreements with other studies or reviews section below.

\section{Risk of bias in included studies}

No studies met the review's inclusion criteria.

\section{Effects of interventions}

No studies met the review's inclusion criteria.

\section{DISCUSSION}

\section{Summary of main results}

After completing a comprehensive search, we identified no RCTs that focussed on the use of standing devices for boys or adults with DMD.

When reviewing the full-text of the 13 excluded studies, the authors (KP, SMCD, and CK) identified a set of inclusion criteria to allow identification of studies which, in our opinion, could potentially inform future trial design. These criteria identified studies that presented experimental data, i.e. where boys or men with DMD experienced different forms of standing devices or a standing device versus control, and studies that included a minimum of one outcome of interest. We found two studies that met these criteria (Taktak 1995; Townsend 2016). We have discussed them narratively below.

\section{Overall completeness and applicability of evidence}

We did not identify any RCTs that met the inclusion criteria for the review. We identified two non-RCTs that reported outcomes of interest, including range of joint movement, bone density, participation and family satisfaction (Taktak 1995; Townsend 2016). Neither study included our other outcomes of interest, namely; bowel function, scoliosis, pain, quality of life, or respiratory function.

\section{Quality of the evidence}

This review did not find any studies that met the inclusion criteria. The current literature surrounding standing frames and orthoses for DMD lacks the robust methods of RCT designs. RCTs are needed to enhance the overall quality of evidence.

\section{Potential biases in the review process}

The review process did not allow for the explicit identification of potential adverse events. The main limitation of this review was the lack of RCTs.

\section{Agreements and disagreements with other studies or reviews}

This review has similar findings to other reviews on the topic of management of DMD. Cheuk2015, a Cochrane Review of surgery for scoliosis, also included no studies due to a lack of RCTs in the area. Our searches identified two non-randomised studies that presented experimental data. The evidence described narratively below is of very low-certainty, due to small sample size and lack of randomisation. It should be interpreted with caution.

\section{Evidence from non-randomised studies}

\section{Study design, participants and interventions}

The studies were non-randomised; one was a within-group comparison (Taktak 1995), the other a case series (Townsend 2016).

Both studies had very few participants: four in Townsend 2016 and 26 in Taktak 1995. The boys participating were aged between 5 and 15 years, which explained the great variability in their stages of disease progression. At the start of each study, there was a mixed presentation of boys who were ambulant and boys who were nonambulant. Neither study reported whether boys had previously received, or were currently receiving, steroid treatment (Townsend 2016; Taktak 1995).

The standing device used in Taktak 1995 was a knee-ankle-foot-orthosis (KAFO), whereas Townsend 2016 studied the effects of using a standing wheelchair.

\section{Outcomes of interest}

Both studies reported a limited number of outcomes which aligned to the specified focus of this review (Taktak 1995; Townsend 2016). However, it was not clear whether the reported outcomes reflected the effects of the standing device, or natural disease progression.

\section{Change in joint range of movement}

Townsend 2016 reported a change in range of joint movement for the four participants after 6 to 20 months' use of a standing wheelchair (total 47 hours to 192 hours). The study reported range of movement for the ankle joint (plantar flexor length), knee (popliteal angle) and hip (Thomas test position) using goniometry over a period of 6.5 to 20 months.

For hip range of movement, three boys showed improvements (ranging from $9^{\circ}$ to $15^{\circ}$ ), with the fourth boy showing a decrease in range of movement (of $16^{\circ}$ ).

For the ankle joint, plantar flexor length was stable in three boys and decreased in one boy. Two of the boys who had a stable ankle joint range of movement had recently undergone surgery for Achilles tendon lengthening.

Knee flexor length improved in two boys (ranging from $1^{\circ}$ to $6^{\circ}$ ) and declined in two boys (ranging from $10^{\circ}$ to $30^{\circ}$ )

Taktak 1995 did not report change in joint movement. 
Pain

Neither study reported pain as an outcome.

\section{Scoliosis}

Neither study recorded scoliosis.

\section{Bone density}

One study measured bone density, both before and after use of a standing wheelchair (47 to 192 hours' use over 6 to 12 months). The study used a DEXA scan at the lumbar spine region for all four participants (Townsend 2016).

Three boys showed a significant decrease in bone density during the study period, and one boy's bone density remained stable. Two boys showed an initial decrease in bone density between baseline and mid-intervention (four months) before returning to baseline levels at the point of intervention withdrawal (eight months). One boy had an initial decrease in lumbar spine bone mineral density from baseline to mid-intervention, which continued to decrease over the study period. This boy was the only participant who lost ambulation during the intervention period.

Taktak 1995 did not report bone density.

\section{Respiratory function}

Neither study recorded respiratory function.

\section{Participation}

One study recorded participation at eight months, using subjective methods (Townsend 2016). Types of enhanced participation included allowing access to eat breakfast with siblings (one participant), accessing the freezer more readily (three participants), ability to access higher shelves (one participant), standing to urinate (one participant) and playing catch with the dog (one participant).

The following factors limited adherence during the study: device malfunction (two participants), vacation (one participant), back pain (one participant), ankle pain (one participant), limited time (two participants), and motivation (four participants). The study reported that standing tolerance within a given session was limited due to plantar foot paraesthesia (one participant), knee or shin discomfort (three participants), low back pain (one participant), foot pressure (one participant) and leg fatigue (four participants).

Taktak 1995 did not report participation measures.

\section{Quality of life}

Neither study reported quality of life.

\section{Bowel function}

Neither study reported bowel function.

\section{Participant and family satisfaction}

One study recorded participant and family satisfaction using a study-specific questionnaire (Taktak 1995).

All eight participants reported that a modular KAFO provided a better cosmetic effect than the traditional KAFO. All participants reported little or no difference in relation to the comfort of the two devices, and five participants reported that they preferred the modular KAFO in terms of weight. Two participants reported increased independence in relation to putting on and taking off the modular KAFO, in comparison to the traditional devices.

\section{Adverse events}

Neither study reported any adverse events.

\section{Standard of care guidelines}

The standard of care guidelines for Duchenne muscular dystrophy have recently been updated (Birnkrant 2018a; Birnkrant 2018b). These standards are based on expert consensus. These guidelines recommend the use of passive or motorised supported standing devices for prevention of contracture and deformity when standing in good alignment becomes difficult, and if contractures are not too severe to prevent positioning or tolerance (Birnkrant 2018a). Standing devices are also recommended in the early non-ambulatory stage to initiate a standing programme. The guidelines recommend using standing programmes with caution in the late non-ambulatory stage (Birnkrant 2018b).

\section{AUTHORS' CONCLUSIONS}

\section{Implications for practice}

We lack evidence from randomised controlled trials (RCTs) to evaluate the effectiveness of standing devices in boys and men with Duchenne muscular dystrophy.

\section{Implications for research}

We need further studies to investigate the effectiveness of standing devices in this population. Due to the ethical considerations of removing a standing device, it may be more appropriate to randomise to different types of standing devices. It would also be possible to use randomised controlled trial methods to determine the optimal 'dose' of standing device use, and investigate the optimum age range for the intervention. Other studies should investigate strategies to increase adherence and maintain motivation for standing programmes. Future studies should use valid and reliable outcome measures that are clinically relevant. Future studies should also include carefully designed control groups to determine which elements of the intervention are influencing the outcome, i.e. the standing device or other therapy techniques.

\section{ACKNOWLEDGEMENTS}

The review authors would like to acknowledge the Research and Development Division of the Public Health Agency (Northern Ireland) for their funding of the Cochrane Fellowship for Katy Pedlow. The Cochrane Neuromuscular Information Specialist, Angela Gunn, developed search strategies in collaboration with the review authors.

This project was supported by the National Institute for Health Research via Cochrane Infrastructure funding to Cochrane Neuromuscular. The views and opinions expressed therein are those of the authors and do not necessarily reflect those of the Systematic Reviews Programme, NIHR, NHS or the Department of Health. Cochrane Neuromuscular is also supported by the MRC Centre for Neuromuscular Disease. 


\section{RE F E R E N C E S}

\section{References to studies excluded from this review}

\section{Alemdaroglu 2014 \{published data only\}}

Alemdaroglu I, Gur G, Bek N, Yilmaz O, Yakut Y, Uygur F, et al. Is there any relationship between orthotic usage and functional activities in children with neuromuscular disorders?. Prosthetics and Orthotics International 2013;38(1):27-33.

\section{Bakker 1997 \{published data only\}}

Bakker JP, de Groot IJ, de Jong BA, van Tol-de Jager MA, Lankhorts GJ. Prescription pattern for orthoses in the Netherlands: use and experience in the ambulatory phase of Duchenne muscular dystrophy. Disability and Rehabilitation 1997;19(8):318-25.

\section{Bakker 2002 \{published data only\}}

Bakker JP, de Groot IJ, Beelen A, Lankhorst GJ. Predictive factors of cessation of ambulation in patients with Duchenne muscular dystrophy. American Journal of Physical Medicine and Rehabilitation 2002;81:906-12.

\section{Garralada 2006 \{published data only\}}

Gerralada ME, Muntoni F, Cunniff A, Caneja AD. Knee-anklefoot orthosis in children with Duchenne muscular dystrophy: user views and adjustment. Official Journal of the European Paediatric Neurology Society 2006;10:186-91.

\section{Heckmatt 1985 \{published data only\}}

Heckmatt JZ, Dubowitz V, Hyde SA, Florence J, Gabain AC, Thompson N. Prolongation of walking in Duchenne muscular dystrophy with lightweight orthosis: review of 57 cases. Developmental Medicine and Child Neurology 1985;27:149-54.

\section{Hunt 1981 \{published data only\}}

Hunt AK. Use of knee-ankle-foot orthoses for transfers of nonambulatory boys with Duchenne muscular dystrophy. Physical Therapy 1981;61(1):51.

\section{Katz 2006 \{published data only\}}

Katz DE. The use of ambulatory knee-ankle-foot orthoses in paediatric patients. Journal of Prosthetics and Orthotics 2006;18(3S):192-7.

\section{Kinali 2007 \{published data only\}}

Kinali M, Main M, Eliahoo J, Messina S, Knight RK, Lehovsky J, et al. Predictive factors for the development of scoliosis in Duchenne muscular dystrophy. Official Journal of the European Paediatric Neurology Service 2007;11:160-6.

\section{Main 2006 \{published data only\}}

Main M, Mercuri E, Haliloglu G, Baker R, Kinali M, Muntoni F. Serial casting of the ankles in Duchenne muscular dystrophy: can it be an alternative to surgery?. Neuromuscular Disorders 2007;17:227-30.

\section{Rodillo 1988 \{published data only\}}

Rodillop EB, Fernandez-Bermejo E, Hecmatt JZ, Dubowitz V. Prevention of rapidly progressive scoliosis in Duchenne muscular dystrophy by prolongation of walking with orthoses. Journal of Child Neurology 1988;3:269-74.

Taktak 1995 \{published data only\}

Taktak D, Bowker P. Lightweight, modular knee-anklefoot orthosis for Duchenne muscular dystrophy: design, development, and evaluation. Archives of Physical Medicine and Rehabilitation 1995;76:1156-62.

\section{Townsend 2016 \{published data only\}}

Townsend EL, Bibeau C, Holmes T. Supported standing in boys with Duchenne muscular dystrophy. Pediatric Physical Therapy 2016;28(3):320-9.

\section{Vignos 1996 \{published data only\}}

Vignos P, Wagner M, Karlinchak B, Katirji B. Evaluation of a Program for Long Term Treatment of Duchenne Muscular Dystrophy. Journal of Bone and Joint Surgery 1996;78:1844-52.

\section{Additional references}

\section{Bakker 2000}

Bakker JP, de Groot IJ, Beckerman H, de Jong BA, Lankhorst GJ. The effects of knee-ankle-foot orthoses in the treatment of Duchenne muscular dystrophy: review of the literature. Clinical Rehabilitation 2000;14(4):343-59.

\section{Birnkrant 2018a}

Birnkrant DJ, Bushby K, Bann CM, Apkon SD, Blackwell A, Brumbaugh $D$, et al. Diagnosis and management of Duchenne muscular dystrophy, part 1: diagnosis, and neuromuscular, rehabilitation, endocrine, and gastrointestinal and nutritional management. Lancet Neurology 2018;17:251-67.

\section{Birnkrant 2018b}

Birnkrant DJ, Bushby K, Bann CM, Alman BA, Apkon SD, Blackwell $A$, et al. Diagnosis and management of Duchenne muscular dystrophy, part 2: respiratory, cardiac, bone health, and orthopaedic management. Lancet Neurology 2018;17:347-61.

\section{Bunnell 1984}

Bunnell WP. An objective criterion for scoliosis screening. Journal of Bone and Joint Surgery. American Volume 1984;66(9):1381-7.

\section{Caulton 2004}

Caulton JM, Ward KA, Alsop CW, Dunn G, Adams JE, Mughal MZ. A randomised controlled trial of standing programme on bone mineral density in non-ambulant children with cerebral palsy. Archives of Diseases in Childhood 2004;89(2):131-5.

\section{Cheuk 2015}

Cheuk DKL, Wong V, Wraige E, Baxter P, Cole A. Surgery for scoliosis in Duchenne muscular dystrophy. Cochrane Database of Systematic Reviews 2015, Issue 10. [DOI: 10.1002/14651858.CD005375.pub4] 


\section{Deconinck 2007}

Deconinck N, Dan B. Pathophysiology of Duchenne muscular dystrophy: current hypotheses. Pediatric Neurology 2007;36(1):1-7.

\section{Dooner 2007}

Dooner R, Keddie A, Lomax D, Melville P, Morrow M, Wotherspoon L. Duchenne Muscular Dystrophy Scottish Physiotherapy Managment Profile. www.apcp.org.uk/Portals/1/ dmd_management_profile.pdf (accessed 19 February 2014).

\section{Eagle 2002}

Eagle M. Report on the Muscular Dystrophy Campaign workshop: exercise in neuromuscular diseases Newcastle, January 2002. Neuromuscular Disorders 2002;12(10):975-83.

\section{Gibson 2009}

Gibson SK, Sprod JA, Maher CA. The use of standing frames for contracture management for nonmobile children with cerebral palsy. International Journal of Rehabilitation Research 2009;32(4):316-23.

\section{Glickman 2010}

Glickman LB, Geigle PR, Paleg GS. A systematic review of supported standing programs. Journal of Pediatric Rehabilitation Medicine 2010;3(3):197-213.

\section{GRADEpro GDT [Computer program]}

McMaster University (developed by Evidence Prime). GRADEpro GDT. Hamilton (ON): McMaster University (developed by Evidence Prime), accessed 16 September 2019.

\section{Higgins 2011}

Higgins JP, Green S, editor(s). Cochrane Handbook for Systematic Reviews of Interventions Version 5.1 (updated March 2011). The Cochrane Collaboration, 2011. Available from handbook.cochrane.org.

\section{Holland 2006}

Holland D, Holland T. Taking a stand. Rehabilitation Management 2006;19(2):44-9.

\section{King 2004}

King G, Law M, King S, Hurley P, Hanna S, Kertoy M, et al. Children's Assessment of Participation and Enjoyment (CAPE) and Preferences for Activities of Children (PAC). San Antonio, TX: Harcourt Assessment, Inc 2004.

\section{Kohler 2009}

Kohler M, Clarenbach CF, Bahler C, Brack T, Russi EW, Block KE. Disability and survival in Duchenne muscular dystrophy. Journal of Neurology, Neurosurgery and Psychiatry 2009;80(3):320-5.

\section{Landgraf 1999}

Landgraf JM. Measuring pediatric outcomes in applied clinical settings: an update about the Child Health Questionnaire (CHQ). www.pro-newsletter.com/images/PDF/qol23_0.pdf (accessed 16 September 2014).

\section{Lo Cascio 2014}

Lo Cascio CM, Latshang TD, Kohler M, Fehr T, Bloch KE. Severe metabolic acidosis in adult patients with Duchenne muscular dystrophy. Respiration 2014;87(6):499-503.

\section{Manzur 2008}

Manzur AY, Kinali M, Muntoni F. Update on the management of Duchenne muscular dystrophy. Archives of Disease in Childhood 2008;93(11):986-90.

\section{Mazess 1990}

Mazess RB, Barden HS, Bisek JP, Hanson J. Dual-energy x-ray absorptiometry for total-body and regional bone-mineral and soft-tissue composition. American Journal of Clinical Nutrition 1990;51(6):1106-12.

\section{McCormack 1988}

McCormack HM, Horne DJ, Sheather S. Clinical applications of visual analogue scales: a critical review. Psycholological Medicine 1988;18(4):1007-19.

\section{Mendell 2012}

Mendell JR, Shilling C, Leslie ND, Flanigan KM, al-Dahhak R, Gastier-Foster J, et al. Evidence-based path to newborn screening for Duchenne muscular dystrophy. Annals of Neurology 2012;71(3):304-13.

\section{Paleg 2008}

Glickman LB, Geigle PR, Paleg GS. A systematic review of supported standing programs. Journal of Pediatric Rehabilitation Medicine: An Interdisciplinary Approach 2010;3:197-213.

\section{Pandya 1985}

Pandya S, Florence JM, King WM, Robison JD, Oxman M, Province MA, et al. Reliability of goniometric measurements in patients with Duchenne muscular dystrophy. Physical Therapy 1985;65(9):1339-42.

\section{Phillips 2001}

Phillips MF, Quinlivan RC, Edwards RH, Calverley PM. Changes in spirometry over time as a prognostic marker in patients with Duchenne muscular dystrophy. American Journal of Respiratory and Critical Care Medicine 2001;164(12):2191-4.

\section{Pin 2007}

Pin TW. Effectiveness of static weight-bearing exercises in children with cerebral palsy. Pediatric Physical Therapy 2007;19(1):62-73.

\section{Pope 2007}

Pope PM. Severe and Complex Neurological Disability: Management of the Physical Condition. London: Elsevier, 2007.

\section{Revman 2014 [Computer program]}

Nordic Cochrane Centre, The Cochrane Collaboration. Review Manager 5 (Revman 5). Version 5.3. Copenhagen: Nordic Cochrane Centre, The Cochrane Collaboration, 2014. 


\section{Rivi 2014}

Rivi E, Filippi M, Fornasari E, Mascia MT, Ferrari A, Costi S. Effectiveness of standing frame on constipation in children with cerebral palsy: a single-subject study. Occupational Therapy International 2014;21(3):115-23.

\section{Taylor 2009}

Taylor K. Factors affecting prescription and implementation of standing-frame programs by school-based physical therapists for children with impaired mobility. Pediatric Physical Therapy 2009;21(3):282-8.

\section{Umphred 2013}

Hallum A, Allen DD. Neuromuscular diseases. In: Umphred DA, Lazaro RT, Roller ML, Burton GU editor(s). Umphred's Neurological Rehabilitation. 6th Edition. St Louis: Elsevier, Mosby, 2013:521-70.

\section{CHARACTERISTICS OF STUDIES}

Characteristics of excluded studies [ordered by study ID]

\section{Vincent 2007}

Vincent KA, Carr AJ, Walburn J, Scott DL, Rose MR. Construction and validation of a quality of life questionnaire for neuromuscular disease (INQoL). Neurology 2007;68(13):1051-7.

\section{Wills 2007}

Wills BP, Auerbach JD, Zhu X, Caird MS, Horn BD, Flynn JM, et al. Comparison of Cobb angle measurement of scoliosis radiographs with preselected end vertebrae: traditional versus digital acquisition. Spine 2007;32(1):98-105.

\section{References to other published versions of this review Pedlow 2015 \\ Pedlow K, McDonough S, Lennon S, Kerr C, Bradbury I. Assisted standing for Duchenne muscular dystrophy. Cochrane Database of Systematic Reviews 2015, Issue 3. [DOI: 10.1002/14651858.CD011550]}

\section{Study Reason for exclusion $\begin{array}{ll}\text { Alemdaroglu } 2014 & \text { Methodology and design not eligible. A retrospective cohort study investigating the relationship } \\ \text { between orthosis use and current functional level. }\end{array}$ \\ Bakker 1997 \\ Methodology and design not eligible. A cohort study that reviewed the orthoses prescription pat- tern by physicians and evaluated the patient experience of orthosis use.}

Bakker 2002

Methodology and design not eligible. A prospective cohort study that investigated predictive factors for loss of ambulation including muscle strength, range of motion, and scoliosis.

\section{Garralada $2006 \quad$ Methodology and design not eligible. A qualitative study that explored the views and the adjust-} ment of families in relation to the use of KAFOs

Meckmatt 1985 Methodology and design not eligible. A retrospective cohort study that examined the impact of
providing a lightweight ankle-foot orthosis on walking prolongation.

Hunt 1981 Methodology and design not eligible. A description of how to transfer a boy with DMD whilst a KAFO was being used.

Katz 2006

Methodology and design not eligible. A review paper that evaluated the evidence for using ambulatory KAFOs in children, including those with DMD.

Kinali 2007

Methodology and design not eligible. A retrospective cohort study that evaluated predictive factors for the development of scoliosis, including prolonged ambulated and glucocorticoid administration.

Main 2006 Methodology and design not eligible. A cohort study that investigated the difference in outcome
between serial casting and surgery in relation to Achilles tendon length.
habilitation on the development of scoliosis. 


\begin{tabular}{ll}
\hline Study & Reason for exclusion \\
\hline Townsend 2016 & $\begin{array}{l}\text { Methodology and design not eligible. This study used a case series design to determine the safety, } \\
\text { efficacy and tolerability of standing devices for boys with DMD, focusing specifically muscle length } \\
\text { and bone density. }\end{array}$ \\
\hline Vignos 1996 & $\begin{array}{l}\text { Methodology and design not eligible. This retrospective cohort study evaluated the long term value } \\
\text { of physical therapy operative treatment and bracing in the control of contractures. }\end{array}$
\end{tabular}

DMD: Duchenne muscular dystrophy

KAFO: knee ankle foot orthosis

\section{AP PENDICES}

\section{Appendix 1. Cochrane Neuromuscular Specialised Register (CRSWeb) search strategy}

Search date: 5 September 2019

\#1 duchenne AND INREGISTER

\#2 standing NEAR3 frame* AND INREGISTER

\#3 standing NEAR3 device* AND INREGISTER

\#4 (stand or standing) NEAR3 support* AND INREGISTER

\#5 stander AND INREGISTER

\#6 standing NEAR3 wheelchair ${ }^{\star}$ AND INREGISTER

\#7 (stand or standing) NEAR3 aid* AND INREGISTER

\#8 kafo* AND INREGISTER

\#9 knee ankle foot ortho* AND INREGISTER

\#10 assist* NEAR3 stand* AND INREGISTER

\#11 loss NEAR3 stand ${ }^{\star}$ AND INREGISTER

\#12 tilt $^{\star}$ table ${ }^{*}$ AND INREGISTER

\#13 "orthotic device" or "orthotic devices" or brace or braces AND INREGISTER

\#14 \#13 and (stand or standing) AND INREGISTER

$\# 15$ \#2 or \#3 or \#4 or \#5 or \#6 or \#7 or \#8 or \#9 or \#10 or \#11 or \#12 or \#14 AND INREGISTER

\#16 \#1 and \#15 AND INREGISTER

\section{Appendix 2. Cochrane central Register of Controlled Studies (CENTRAL) (CRSWeb) search strategy}

Search date 5 September 2019

\#1 duchenne AND CENTRAL:TARGET

\#2 standing NEAR3 frame* AND CENTRAL:TARGET

\#3 standing NEAR3 device* AND CENTRAL:TARGET

\#4 (stand or standing) NEAR3 support* AND CENTRAL:TARGET

\#5 stander AND CENTRAL:TARGET

\#6 standing NEAR3 wheelchair ${ }^{\star}$ AND CENTRAL:TARGET

\#7 (stand or standing) NEAR3 aid ${ }^{\star}$ AND CENTRAL:TARGET

\#8 kafo* AND CENTRAL:TARGET

\#9 knee ankle foot ortho* AND CENTRAL:TARGET

$\# 10$ assist* NEAR3 stand ${ }^{*}$ AND CENTRAL:TARGET

\#11 loss NEAR3 stand* AND CENTRAL:TARGET

\#12 tilt* table* AND CENTRAL:TARGET

\#13 "orthotic device" or "orthotic devices" or brace or braces AND CENTRAL:TARGET

\#14 \#13 and (stand or standing) AND CENTRAL:TARGET

$\# 15 \# 2$ or \#3 or \#4 or \#5 or \#6 or \#7 or \#8 or \#9 or \#10 or \#11 or \#12 or \#14 AND CENTRAL:TARGET

$\# 16 \# 1$ and \#15 AND CENTRAL:TARGET

\section{Appendix 3. MEDLINE (OvidSP) search strategy}

Database: Ovid MEDLINE(R) and Epub Ahead of Print, In-Process \& Other Non-Indexed Citations and Daily (1946 to September 04, 2019) Search Strategy: 
1 randomized controlled trial.pt. (488652)

2 controlled clinical trial.pt. (93265)

3 randomi\#ted.tw. (583721)

4 placebo.ab. (200473)

5 drug therapy.fs. (2136487)

6 randomly.ab. (317408)

7 trial.ab. (475229)

8 groups.ab. (1949928)

9 or/1-8 (4540624)

10 exp animals/ not humans.sh. (4615097)

119 not 10 (3933179)

12 Muscular Dystrophy, Duchenne/ (5059)

13 duchenne.tw. (10686)

14 or/12-13 (11618)

15 (standing adj3 frame\$).mp. (127)

16 (standing adj3 device\$).mp. (131)

17 ((stand or standing) adj3 support\$2).mp. (647)

18 stander.mp. (111)

19 (standing adj3 wheelchair\$1).mp. (29)

20 (stand\$3 adj3 aid\$1).mp. (365)

21 kafo\$1.mp. (82)

22 knee ankle foot ortho\$.mp. (199)

23 (assist\$ adj3 stand\$3).mp. (761)

24 (loss adj3 stand\$3).mp. (739)

25 tilt\$ table\$.mp. (3291)

26 Orthotic Devices/ (6250)

27 braces/ (5334)

28 brace $\$ 1 . t w$. (6343)

2926 or 27 or 28 (14911)

3029 and (stand or standing).mp. (568)

31 or/15-25,30 (6795)

3211 and 14 and $31(6)$

3314 and 31 (30)

34 remove duplicates from 33 (30)

\section{Appendix 4. Embase (OvidSP) search strategy}

Database: Embase (1974 to 2019 September 04)

Search Strategy:

1 crossover-procedure.sh. (60577)

2 double-blind procedure.sh. (165194)

3 single-blind procedure.sh. (36475)

4 randomized controlled trial.sh. (568805)

5 (random $\$$ or crossover\$ or cross over\$ or placebo $\$$ or (doubl\$ adj blind\$) or allocat\$).tw,ot. (1685633)

6 trial.ti. (280325)

7 or/1-6 (1863745)

8 exp animal/ or exp invertebrate/ or animal.hw. or non human/ or nonhuman/ (26434028)

9 human/ or human cell/ or human tissue/ or normal human/ (20194559)

108 not 9 (6303486)

117 not 10 (1643351)

12 limit 11 to (conference abstracts or embase) (1380034)

13 Duchenne muscular dystrophy/ (15436)

14 Duchenne.tw. (14836)

1513 or $14(18690)$

16 (standing adj3 frame\$).mp. (229)

17 (standing adj3 device\$).mp. (139)

18 (standing adj3 support\$2).mp. (599)

19 stander.mp. (259)

20 exp foot orthosis/ (2978)

21 orthotics/ (3685)

22 brace/ (8859) 
23 (or/20-22) and (stand or standing).mp. (955)

24 or/16-19,23 (2089)

2512 and 15 and 24 (1)

2615 and 24 (20)

27 remove duplicates from $26(20)$

28 limit 27 to (conference abstracts or embase) (18)

\section{Appendix 5. CINAHL Plus (EBSCOhost) search strategy}

Friday, September 06, 2019 10:36:25 AM

S41 S20 and S37 12

S40 S38 AND S39 1

S39 EM 20180301-635,649

S38 S19 AND S20 AND S37 5

S37 S21 OR S22 OR S23 OR S24 OR S25 OR S26 OR S27 OR S28 OR S29 OR S30 OR S31 OR S32 OR S36 5,286

S36 S35 and (stand or standing) 891

S35 S33 OR S34 13,625

S34 brace or braces 2,421

S33 orthotic device* or orthos* 12,564

S32 tilt* table* 908

S31 loss N3 stand 368

S30 assist ${ }^{\star} \mathrm{N} 3$ stand $^{\star} 1,814$

S29 knee ankle foot ortho* 152

S28 kafo* 59

S27 stand* N3 aid* 838

S26 standing N3 wheelchair* 27

S25 stander 52

S24 (stand or standing) N3 support* 275

S23 standing N3 device* 56

S22 standing N3 frame* 71

S21 standing frames 67

S20 Duchenne and dystroph ${ }^{\star} 1,897$

$\mathrm{S} 19 \mathrm{~S} 2$ or S3 or S4 or S5 or S6 or S7 or S8 or S9 or S10 or S11 or S12 or S13 or S14 or S15 or S16 or S17 or S18 1,264,592

S18 ABAB design* 121

S17 TI random* or AB random* 299,617

S16 ( TI (cross?over or placebo* or control* or factorial or sham? or dummy) ) or ( AB (cross?over or placebo* or control* or factorial or sham? or dummy) ) 597,547

S15 ( $\mathrm{TI}$ (clin* or intervention* or compar ${ }^{\star}$ or experiment ${ }^{\star}$ or preventive or therapeutic) or AB (clin* or intervention* or compar ${ }^{\star}$ or experiment $^{\star}$ or preventive or therapeutic) ) and ( $\mathrm{TI}\left(\right.$ trial $\left.l^{\star}\right)$ or AB $\left(\right.$ trial $\left.^{\star}\right)$ ) 231,767

S14 ( TI (meta?analys* or systematic review*) ) or ( AB (meta?analys* or systematic review*) ) 85,194 


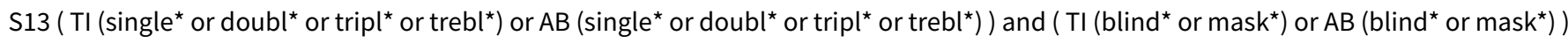
45,616

S12 PT ("clinical trial" or "systematic review") 166,169

S11 (MH "Factorial Design") 1,139

S10 (MH "Concurrent Prospective Studies") or (MH "Prospective Studies") 393,415

S9 (MH "Meta Analysis") 39,710

S8 (MH "Solomon Four-Group Design") or (MH "Static Group Comparison") 98

S7 (MH "Quasi-Experimental Studies") 11,183

S6 (MH "Placebos") 11,443

S5 (MH "Double-Blind Studies") or (MH "Triple-Blind Studies") 42,452

S4 (MH "Clinical Trials+") 266,130

S3 (MH "Crossover Design") 17,909

S2 (MH "Random Assignment") or (MH "Random Sample") or (MH "Simple Random Sample") or (MH "Stratified Random Sample") or (MH "Systematic Random Sample") 93,606

S1 (MH "Crossover Design") 17,909

\section{Appendix 6. AMED (OvidSP) search strategy}

Database: AMED (Allied and Complementary Medicine) (1985 to August 2019)

Search Strategy:

1 Randomized controlled trials/ (2251)

2 Random allocation/ (323)

3 Double blind method/ (732)

4 Single-Blind Method/ (137)

5 exp Clinical Trials/ (4110)

6 (clin\$ adj25 trial\$).tw. (7582)

7 ((singl\$ or doubl\$ or treb\$ or trip\$) adj25 (blind\$ or mask\$ or dummy)).tw. (3187)

8 placebos/ (626)

9 placebo\$.tw. (3396)

10 random $\$ . t w .(19575)$

11 research design/ (2003)

12 Prospective Studies/ (1320)

13 meta analysis/ (299)

14 (meta?analys\$ or systematic review\$).tw. (4280)

15 control\$.tw. (38515)

16 (multicenter or multicentre).tw. (1127)

17 ((study or studies or design\$) adj25 (factorial or prospective or intervention or crossover or cross-over or quasi-experiment\$)).tw. (14048)

18 or/1-17 (59676)

19 (muscular dystrophy and duchenne).mp. (245)

20 (standing adj3 frame\$).mp. (28)

21 (standing adj3 device\$).mp. (26)

22 ((stand or standing) adj3 support\$2).mp. (72)

23 stander.mp. (2)

24 (standing adj3 wheelchair\$1).mp. (10)

25 (stand\$3 adj3 aid\$1).mp. (16)

26 kafo\$1.mp. (31)

27 knee ankle foot ortho\$.mp. (65)

28 (assist\$ adj3 stand\$3).mp. (67)

29 (loss adj3 stand\$3).mp. (20)

30 tilt\$ table\$.mp. (47)

31 exp orthotic devices/ (2118)

32 brace $\$ 1 . m p$. (759) 
3331 or 32 (2502)

3433 and (stand or standing).mp. (153)

35 or/20-30,34 (457)

3618 and 19 and 35 (3)

3719 and 35 (8)

\section{Appendix 7. PsycINFO (OvidSP) search strategy}

Database: PsycINFO (1806 to August Week 4 2019)

Search Strategy:

1 (random\$ or rct or cct or factorial\$ or crossover\$ or cross over\$ or cross-over\$ or placebo\$ or (doubl\$ adj blind\$) or (singl\$ adj blind\$) or assign $\$$ or allocat\$ or volunteer\$).tw. (341095)

2 (duchenne adj5 dystroph\$).mp. (632)

3 (standing adj3 frame\$).mp. (31)

4 (standing adj3 device\$).mp. (9)

5 ((stand or standing) adj3 support\$2).mp. (175)

6 stander.mp. (28)

7 (standing adj3 wheelchair\$1).mp. (7)

8 (stand\$3 adj3 aid\$1).mp. (78)

9 kafo\$1.mp. (2)

10 knee ankle foot ortho\$.mp. (3)

11 (assist\$ adj3 stand\$3).mp. (92)

12 (loss adj3 stand\$3).mp. (126)

13 tilt\$ table\$.mp. (187)

14 orthotic device\$.mp. (174)

15 brace $\$ 1 . m p$. (343)

1614 or 15 (513)

1716 and (stand or standing).mp. (32)

18 or/4-13,17 (727)

191 and 2 and 18 (0)

202 and $18(0)$

\section{Appendix 8. ProQuest Dissertations \& Theses Global search strategy}

Search date 5 September 2019

all(standing OR stand OR stander) AND all(duchenne) AND all(KAFO OR "knee ankle foot orthosis" OR "tilt table" OR "orthotic device*" OR brace OR Braces OR wheelchair) AND (randomized OR randomised OR randomly) = 1 result.

\section{Appendix 9. PEDro search strategy}

Duchenne $=27$ results. 0 selected to follow up based on title and abstract.

\section{Appendix 10. Clinical trial registry databases}

Duchenne and standing and orthosis. 0 results.

\section{Appendix 11. Additional methods}

The following are additional methods as specified in our protocol. We did not employ them in the review owing to the absence of included studies.

\section{Data collection and analysis}

\section{Selection of studies}

Two review authors (KP, SMcD) will independently screen titles and abstracts of all the potential studies we identify as a result of the search for inclusion and code them as 'retrieve' (eligible or potentially eligible/unclear) or 'do not retrieve'. We will identify and exclude duplicates and collate multiple reports of the same study so that each study rather than each report is the unit of interest in the review. We will retrieve full-text study reports, and two review authors (KP, SMcD) will independently screen the full text and identify studies for inclusion, and identify and record reasons for exclusion of the ineligible studies. We will resolve any disagreements through discussion or, if required, through consultation with a third review author (CK). We will report the selection process in sufficient detail to complete a PRISMA flow diagram and 'Characteristics of excluded studies' table. 


\section{Data extraction and management}

We will use a data extraction form to extract study characteristics and outcome data; we will pilot this form on at least one study in the review. Two review authors (KP, CK) will extract study characteristics from included studies. We will note if any outcome data were not reported in a usable way. We will extract the following study characteristics.

1. Methods: study design, total duration of study, details of any 'run in' period, number of study centres and location, study setting, withdrawals and date of study

2. Participants: N, mean age, age range, gender, current residential country, severity of condition, diagnostic criteria, baseline characteristics, inclusion criteria and exclusion criteria

3. Interventions: intervention, number in each intervention group, comparison, concomitant medications and excluded medications

4. Outcomes: primary and secondary outcomes specified and collected and time points reported.

5. Results or main findings: primary and secondary results at all time points. Main findings will also report the number of previous fractures and the age at which scoliosis surgery was completed.

6. Others: conflicts of interest among main investigators and study funding source(s)

We will resolve disagreements by consensus or by involving a third person (SL). One review author (KP) will transfer data into Review Manager (Revman 2014). A second review author will check the outcome data entries (CK). A third review author (SL) will spot-check study characteristics for accuracy against the trial report.

When reports require translation, the translator will extract data directly using a data extraction form, or review authors will extract data from the translation provided. Where possible, a review author will check numerical data in the translation against the study report.

\section{Assessment of risk of bias in included studies}

Two review authors (KP, SL) will independently assess risk of bias for each study using the criteria outlined in the Cochrane Handbook for Systematic Reviews of Interventions (Higgins 2011). These authors will resolve disagreements by discussion or by involving another review author $(\mathrm{SMCD})$.

We will assess the risk of bias according to the following domains: random sequence generation, allocation concealment, blinding of participants and personnel, blinding of outcome assessment, incomplete outcome data, selective outcome reporting and other bias.

We will grade each potential source of bias as high, low, or unclear and provide a quote from the study report together with a justification for our judgment in the 'Risk of bias' table. We will summarise the risk of bias judgements across different studies for each of the domains listed. We will consider blinding separately for different key outcomes where necessary (for example for unblinded outcome assessment, risk of bias for all-cause mortality may be very different than for a patient-reported pain scale). Where information on risk of bias relates to unpublished data or correspondence with a trialist, we will note this in the 'Risk of bias' table. When considering treatment effects, we will take into account the risk of bias for the studies that contribute to that outcome.

\section{Assessment of bias in conducting the systematic review}

We will conduct the review according to this published protocol and report any deviations from it in the 'Differences between protocol and review' section of the systematic review.

\section{Measures of treatment effect}

We will analyse dichotomous data as risk ratios with 95\% confidence interval (Cl) and continuous data as mean difference with $95 \% \mathrm{Cl}$, or standardised mean difference with $95 \% \mathrm{Cl}$ for results across studies with outcomes that are conceptually the same, but measured in different ways. We will enter data presented as a scale with a consistent direction of effect.

We will undertake meta-analyses only where this is meaningful, that is if the treatments, participants and the underlying clinical question are similar enough for pooling to make sense.

We will narratively describe skewed data reported as medians and interquartile ranges.

Where a single trial reports multiple trial arms, we will include only the relevant arms.

\section{Unit of analysis issues}

We do not expect to have any cross-over or cluster-randomised controlled trials, and therefore do not expect any unit-of-analysis issues arising from these designs. If we do include a cross-over study, then, due to the progressive nature of DMD, we will analyse only the first arm if the treatment arm precedes the control. If a study has more than one treatment arm (for example standing frame A versus control and standing frame B versus control), and the study did not combine the active treatment arms for analysis, we will split the control treatment arm between active treatment arms to avoid double-counting. 


\section{Dealing with missing data}

We will contact investigators or study sponsors to verify key study characteristics and to obtain missing numerical outcome data where possible (for example, when a study is available as an abstract only). Where this is not possible, and it is thought that the missing data introduces serious bias, we will use a sensitivity analysis to explore the impact of including such studies in the overall assessment of results. We will report and compare descriptively the characteristics of studies with missing outcome data with the characteristics of complete studies.

\section{Assessment of heterogeneity}

We will use the $\mathrm{I}^{2}$ statistic to measure heterogeneity among the trials in each analysis. If we identify substantial unexplained heterogeneity, we will report both fixed-effect and random-effects results and explore possible causes by prespecified subgroup analysis.

\section{Assessment of reporting biases}

If we are able to pool more than 10 trials, we will create and examine a funnel plot to explore possible small-study biases. To further determine reporting bias, we will request study protocols and use the ORBIT framework to examine the impact of selective reporting bias.

\section{Data synthesis}

We will use a fixed-effect model, providing there is no evidence of heterogeneity of effect. We will use a random-effects model if there is evidence of statistical heterogeneity.

If the review includes more than one comparison that cannot be included in the same analysis, we will report the results for each comparison separately.

\section{'Summary of findings' table}

We will create a 'Summary of findings' table using the following outcomes.

1. Goniometry, change in value from baseline to 12 months

2. Pain (VAS), change in value from baseline to three months

3. Scoliosis (scoliometer), change in value from baseline to over 12 months

4. Bone density (DEXA), change in value from baseline to over 12 months

5. Respiratory function (FVC\%), change in value from baseline to six to nine months

6. Quality of life (INQoL), change in value from baseline to 12 months

7. Adverse events

We will use the five GRADE considerations (study limitations, consistency of effect, imprecision, indirectness, and publication bias) to assess the quality of a body of evidence (studies that contribute data for the prespecified outcomes). We will use methods and recommendations described in Chapter 12 of the Cochrane Handbook for Systematic Reviews of Interventions (Higgins 2011) using GRADEpro software (GRADEpro GDT). We will justify all decisions to down- or upgrade the quality of studies using footnotes, and we will make comments to aid readers' understanding of the review where necessary.

\section{Subgroup analysis and investigation of heterogeneity}

We plan to carry out the following subgroup analyses.

1. Dosage of standing frame or orthoses use.

2. Age of participant ( 0 to 12 years, 13 to 17 years, 18 years plus).

We will use the formal test for subgroup interactions in Review Manager (Revman 2014).

\section{Sensitivity analysis}

We plan to carry out the following sensitivity analyses.

1. Repeat the analysis excluding any unpublished studies.

2. Repeat the analysis excluding studies at high risk of bias.

3. If there are one or more very large studies, repeat the analysis excluding those studies to explore to what degree they dominate the results.

\section{Reaching conclusions}

We will base our conclusions only on findings from the quantitative or narrative synthesis of included studies for this review. We will avoid making recommendations for practice, and our implications for research will suggest priorities for future research and outline the remaining uncertainties in the area. 


\section{CONTRIBUTIONS OF AUTHORS}

All review authors contributed to the writing and reviewing of the protocol.

KP: protocol development, searches for trials, data extraction, development of final review, corresponding author.

SMCD: protocol development, searches for trials, data extraction, development of final review.

CK: protocol development, searches for trials, data extraction, development of final review.

SL: protocol development, development of the final review.

IB: protocol development, development of the final review.

\section{DECLARATIONSOF INTEREST}

IB: none known

CK: received no support from any organisation for the submitted work; she has received a research grant in partnership with Leckey.

SL: none known.

SMCD: none known

KP: was funded by Health and Social Care (HSC) Research and Development Northern Ireland for the completion of this Cochrane Review.

\section{SOURCES OF SUPPORT}

\section{Internal sources}

- No sources of support supplied

\section{External sources}

- The Research and Development Division of the Public Health Agency (Northern Ireland), UK.

Cochrane Fellowship Funding Award for Dr Katy Pedlow

\section{DIFFERENCES BETWEEN PROTOCOLANDREVIEW}

We did not implement the methods specified in our protocol for data extraction and analysis because the review included no eligible studies. We therefore could not complete various methods, including: data extraction, 'Risk of bias' assessment, measurement of treatment effect, assessment of heterogeneity and reporting bias, data synthesis or sensitivity analysis. We were unable to complete a 'Summary of findings' table or complete subgroup analysis. Appendix 11 details additional methods planned in the protocol. 